\title{
ATUAÇÃO DO ENFERMEIRO NA CIRURGIA ROBÓTICA: DESAFIOS E PERSPECTIVAS
}

\author{
Nurse role in robotic surgery: challenges and prospects
}

\author{
Actuación del enfermero en la cirugía robótica: retos y perspectivas
}

Elisandra Venzke Pinto' , Liege Segabinazzi Lunardi², Patrícia Treviso ${ }^{3 *}$, Daisy Zanchi de Abreu Botene ${ }^{4}$

RESUMO: Objetivo: Identificar os principais desafios e perspectivas da atuação do enfermeiro na cirurgia robótica. Método: Revisão integrativa, realizada nas bases de dados Literatura Latino-Americana e do Caribe em Ciências da Saúde (LILACS), PubMed e ScienceDirect, de artigos publicados entre 2008 e abril de 2015; amostra constituída por 11 artigos. Para análise dos dados, realizou-se síntese das informações extraídas conforme o objetivo, buscando identificar as evidências. Resultados: A busca resultou na identificação de 281 estudos, sendo 225 na ScienceDirect, 51 na PubMed e cinco na LILACS. Foram selecionados 11 estudos que atendiam aos critérios de inclusão e estavam alinhados à questão norteadora, sendo 9 qualitativos, 1 revisão sistemática de ensaio clínico randomizado e 1 estudo de validação. Conclusão: Evidenciaram-se como desafios: segurança do paciente, capacitação da equipe e desenvolvimento de competências. Em relação às possibilidades, observaram-se uma nova área de atuação em desenvolvimento, a importância da educação permanente e da inovação para a melhoria no atendimento aos pacientes cirúrgicos.

Palavras-chave: Robótica. Enfermagem. Sala de cirurgia. Procedimento cirúrgico. Procedimento cirúrgico minimamente invasivo.

ABSTRACT: Objective: To identify the main challenges and prospects of the nurse's role in robotic surgery. Method: Integrative review carried out in the Literatura Latino-Americana e do Caribe em Ciências da Saúde (LILACS), PubMed and Science Direct databases, of articles published between 2008 and April 2015; sample consisted of 11 articles. The data analysis was carried out by synthesizing the information obtained according to the objective, seeking to identify evidences. Results: The search resulted in the identification of 281 studies, 225 in ScienceDirect, 51 in PubMed and 5 in LILACS. Eleven studies meeting the inclusion criteria and the guiding questioning presented here were selected, being 9 qualitative ones, 1 systematic review of randomized clinical trial and 1 validation study. Conclusion: The challenges presented were: patient safety, team training and skills development. Regarding the possibilities, a new area of development, the importance of permanent education and innovation for the improvement of care of surgical patients were observed. Keywords: Robotics. Nursing. Operating rooms. Surgical procedures, operative. Minimally invasive surgical procedures.

RESUMEN: Objetivo: Identificar los principales desafiós y perspectivas de la actuación del enfermero en la cirugía robótica. Método: Revisión integrative, llevada a cabo en las bases de datos Literatura Latinoamericana y del Caribe en Ciências de la Salud (LILACS), PubMed y Science Direct, de artículos publicados entre 2008 y abril de 2015; muestra constituida por 11 artículos. Para el análisis de losdatos, se realize una síntesis de las informaciones extraídas conforme el objetivo, buscando identificar las evidencias. Resultados: La búsqueda result en la identificación de 281 estudios, 225 en la ScienceDirect, 51 en PubMed y 5 en LILACS. Se seleccionaron 11 estudios que atendían a los criterios de inclusion y estaban alineados a la ceustión orientadora, siendo 9 cuaitativos, 1 revisión sistemática de ensayo clínico randomizado y 1 estudio de validación. Conclusión: Se evidenció como desafios: seguridad del paciente, capacitación del equipo y desarollo de competencias. En cuanto a las posibilidades, se observó una nueva área de actuación en desarollo, la importancia de la educación permanente y de la innovación para la mejora en la atención a los pacientes quirúrgicos.

Palabras clave: Robótica. Enfermería. Quirófanos. Procedimientos quirúrgicos operativos. Procedimientos quirúrgicos mínimamente invasivos.

\footnotetext{
'Enfermeira; pós-graduada em Unidade de Terapia Intensiva, Hospital Mãe de Deus - Porto Alegre (RS), Brasil.

${ }^{2}$ Enfermeira; pós-graduada em Centro Cirúrgico; Hospital de Clínicas de Porto Alegre - Porto Alegre (RS), Brasil.

${ }^{3}$ Enfermeira; doutora em Ciências da Saúde, Centro Universitário Metodista IPA - Porto Alegre (RS), Brasil.

¿Enfermeira; doutora em Enfermagem, Centro Universitário Metodista IPA - Porto Alegre (RS), Brasil.

*Autor correspondente: ptreviso15@gmail.com

Recebido: 31/08/2017 - Aprovado: 09/12/2017

DOl: $10.5327 / Z 1414-4425201800010008$
} 


\section{INTRODUÇão}

A cirurgia robótica tem sido foco de atenção na atualidade por sua relevância na área da saúde. Tanto na área da prevenção quanto na da reabilitação, em diversos segmentos, observa-se o aumento da utilização da tecnologia, fundamental na área da saúde e um desafio para os profissionais de enfermagem, que devem se manter atualizados, considerando a fugacidade da evolução tecnológica ${ }^{1}$.

$\mathrm{O}$ avanço da tecnologia pode proporcionar benefícios à enfermagem quando possibilita ao profissional relacionar o todo e as partes com base nas informações, nos acontecimentos e nos conhecimentos disponibilizados, favorecendo o pensamento unificador e a integração dos elementos a partir da reunião de informações múltiplas que, interligadas, oportunizam associações e interpretações das partes para a compreensão do paciente. Assim, pode-se dizer que o ser humano seria melhor compreendido e cuidado na sua complexidade com o auxílio dessas tecnologias, se considerados tais aspectos ${ }^{2}$.

Quando a tecnologia é adequadamente utilizada e administrada, beneficia a prática do cuidado em diversas áreas ${ }^{3}$. Um exemplo é a unidade de centro cirúrgico (CC), onde tem ocorrido o aumento exponencial de complexidade tecnológica e científica, fazendo com que os pacientes usufruam vários benefícios com o implemento dessas novas formas de atendimento ${ }^{4}$. Nesse contexto de renovação, mudanças, conhecimento e princípios científicos, inclui-se a cirurgia robótica, um novo modelo de cirurgia videolaparoscópica.

Esse modelo inovador busca realizar os procedimentos com reduzido trauma operatório e rápida recuperação póscirúrgica, refletindo em menor morbidade e com reflexo direto no bem-estar dos pacientes. Como vantagens, ressaltam-se: realização de procedimentos de alta complexidade de modo mais simples e prático; redução na agressão aos órgãos e sistemas; facilidade de suturas consideradas difíceis, principalmente em pacientes obesos; e comodidade para o cirurgião, pois propicia operar com ergonomia adequada e de forma confortável, trazendo mais tranquilidade ao profissional na realização de procedimentos mais desgastantes ${ }^{5-7}$.

No Brasil, esse tipo de cirurgia foi implementado em 2008, porém atualmente ainda há poucos sistemas instalados em nosso país, por conta do elevado valor de aquisição e dos insumos. Soma-se a isso o escasso número de empresas que trabalham com essa tecnologia, refletindo na concorrência e, consequentemente, nos preços e custos de implantação ${ }^{6}$. Além das questões financeiras, implementar essa nova tecnologia em um CC exige muito empenho, inúmeras reformas físicas, treinamento das equipes e dos demais profissionais participantes, além de diversos recursos e modificações gerais.

Impõe-se, por conseguinte, a necessidade premente de atualização dos enfermeiros para se tornarem eficazes e sintonizados com as novas exigências do mercado de trabalho que têm se instaurado ${ }^{8}$, permitindo utilizar o que há de mais moderno para assegurar ao paciente a reabilitação de sua saúde e seu bem-estar de modo mais rápido, seguro e eficaz ${ }^{9}$.

\section{OBJETIVO}

Identificar os principais desafios e perspectivas da atuação do enfermeiro na cirurgia robótica.

\section{MÉTODO}

Este estudo é caracterizado como revisão integrativa cuja metodologia consiste em seis etapas: formulação do problema, busca na literatura, coleta de dados, análise crítica dos estudos incluídos, discussão e apresentação dos resultados. Seguindo as etapas, definiu-se como questão norteadora: quais são os desafios e as perspectivas da atuação do enfermeiro na cirúrgica robótica?

Os critérios de inclusão para seleção dos artigos foram: artigos com publicação nos idiomas português do Brasil, inglês ou espanhol, a partir de 2008 — período em que a cirurgia robótica foi implantada no Brasil - e disponíveis na íntegra em meio online. Foram excluídas do estudo publicações em livros, teses e artigos que não abordaram a questão norteadora, ou com textos incompletos.

A pesquisa foi realizada nas bases de dados PubMed, Literatura Latino-Americana e do Caribe em Ciências da Saúde (LILACS) e ScienceDirect, utilizando-se os descritores: robótica, enfermagem de centro cirúrgico, procedimento cirúrgico, procedimentos cirúrgicos minimamente invasivos e enfermagem.

Primeiramente, realizou-se a leitura dos títulos e dos resumos dos artigos encontrados. Posteriormente, os estudos pré-selecionados, ou seja, os artigos que estavam de acordo com os critérios de inclusão e respondiam à questão norteadora, foram lidos criteriosamente na íntegra.

A coleta de dados aconteceu em abril de 2015, por meio de um instrumento contendo: títulos, autores, periódicos, ano de publicação, objetivos, método, resultados e conclusões com o intuito de possibilitar a organização dos estudos selecionados e viabilizar a fase analítico-descritiva. 
Para análise e interpretação dos dados, fez-se a síntese das informações extraídas dos artigos selecionados na elegibilidade, buscando identificar as evidências disponíveis, de acordo com o Centro de Medicina Baseada em Evidências de Oxford ${ }^{10}$, de modo a analisar os resultados encontrados conforme o objetivo traçado.

Este estudo segue os aspectos éticos, em conformidade com os direitos autorais, segundo a Lei $\mathrm{n}^{\mathrm{o}} 9.610 / 98^{11}$.

\section{RESULTADOS}

A busca resultou na identificação de 281 estudos, distribuídos da seguinte forma: ScienceDirect (225), PubMed (51) e
LILACS (5). Foram selecionados, para fazer parte da amostra, 11 estudos que atendiam aos critérios de inclusão e estavam alinhados à questão norteadora, sendo 9 estudos de caráter qualitativo, 1 revisão sistemática de ensaios clínicos randomizados e 1 estudo metodológico de validação. As publicações excluídas referiam-se a publicações repetidas nas bases de dados ou que não respondiam à questão de pesquisa, ou ainda que estavam em desacordo com os critérios de inclusão.

Para facilitar a apresentação dos resultados e a discussão, cada estudo selecionado recebeu um código (de A1 a A11), servindo de base para ser referenciado ao longo da análise. Os estudos incluídos na revisão estão apresentados no Quadro 1.

Quadro 1. Síntese dos estudos incluídos na revisão integrativa de acordo com ano, título, autor(es), periódico e nível de evidência.

\begin{tabular}{|c|c|c|c|c|c|}
\hline Estudo/ano & Título & Autor(es) & Periódico & Tipo de estudo & $\begin{array}{l}\text { Nível de } \\
\text { Evidência }\end{array}$ \\
\hline$A 1^{12} 2009$ & $\begin{array}{c}\text { Technological advances in the } \\
\text { operating room }\end{array}$ & Schmock BA & $\begin{array}{c}\text { Pennsylvania Nurse } \\
\text { Journal }\end{array}$ & Qualitativo & 5 \\
\hline$A 2^{13} 2010$ & Robotics in nursing & Booth BE & $\begin{array}{l}\text { Journal of Nursing } \\
\text { Practice }\end{array}$ & Qualitativo & 5 \\
\hline$A 3^{14} 2010$ & $\begin{array}{l}\text { Best practices for minimally } \\
\text { invasive procedures }\end{array}$ & Ulmer BC & AORN Journal & Qualitativo & 5 \\
\hline$A 4^{15} 2010$ & $\begin{array}{c}\text { The effects of } \\
\text { information technology on } \\
\text { perioperative nursing }\end{array}$ & Sweeney P & AORN Journal & Qualitativo & 5 \\
\hline$A 5^{16} 2010$ & $\begin{array}{l}\text { Developing a successful } \\
\text { robotic surgery program } \\
\text { in a rural hospital }\end{array}$ & Zender J, Thell C & AORN Journal & Estudo de caso & 5 \\
\hline$A 6^{17} 2011$ & Keeping up with technology & Stanton C & AORN Journal & Qualitativo & 5 \\
\hline$A 7^{18} 2011$ & $\begin{array}{l}\text { Role of the perioperative nurse in } \\
\text { robotic surgery }\end{array}$ & Thomas CC & $\begin{array}{l}\text { Perioperative Nursing } \\
\text { Clinics }\end{array}$ & Qualitativo & 5 \\
\hline$A 8^{19} 2011$ & $\begin{array}{l}\text { Robotic-assisted surgery and the } \\
\text { need for standardized pathways } \\
\text { and clinical guidelines }\end{array}$ & Walters L, Eley S & AORN Journal & $\begin{array}{l}\text { Revisão } \\
\text { sistemática } \\
\text { de estudos } \\
\text { randomizados }\end{array}$ & 2 \\
\hline A920 2013 & $\begin{array}{c}\text { Positioning injuries associated } \\
\text { with robotic assisted } \\
\text { urological surgery }\end{array}$ & $\begin{array}{l}\text { Mills JT, Burris } \\
\text { MB, Warburton } \\
\text { DJ, Conaway MR, } \\
\text { Schenkman NS, } \\
\text { Krupski TL }\end{array}$ & Journal of Urology & Estudo de caso & 5 \\
\hline $\mathrm{A} 10^{21} 2013$ & $\begin{array}{l}\text { Modifiable factors to } \\
\text { decrease the cost of } \\
\text { robotic-assisted procedures }\end{array}$ & $\begin{array}{l}\text { Nayeemuddi NM, } \\
\text { Daley SC, Ellsworth P }\end{array}$ & AORN Journal & Qualitativo & 5 \\
\hline$A 11^{22} 2013$ & $\begin{array}{l}\text { Evaluating the influence of } \\
\text { perceived organizational learning } \\
\text { capability on user acceptance of } \\
\text { information technology among } \\
\text { operating room nurse staff }\end{array}$ & $\begin{array}{l}\text { Lee CC, Lin SP, Yang } \\
\text { SL, Tsou MY, Chang KY }\end{array}$ & $\begin{array}{c}\text { Acta Anaesthesiologica } \\
\text { Taiwanica }\end{array}$ & $\begin{array}{l}\text { Quantitativo de } \\
\text { validação }\end{array}$ & 4 \\
\hline
\end{tabular}

AORN: Association of periOperative Registered Nurses. 
A frequência das publicações nos respectivos periódicos é descrita na Tabela 1.

A frequência do nível de evidência das publicações utilizadas está representada na Tabela 2 .

A partir do compilado de artigos revisados, foi possível elencar dois temas principais, contemplando uma síntese do conhecimento em relação à temática no período de estudo:

1. Desafios enfrentados pelo enfermeiro na cirurgia robótica;

2. Perspectivas e possibilidades nesse novo campo de atuação para o enfermeiro.

\section{DISCUSSÕES}

Em relação ao ano de publicação, na fase exploratória, observou-se que as publicações se mantinham regulares desde 2008 , embora no ano de 2012 não se tenha encontrado nenhum

Tabela 1. Distribuição dos estudos conforme o periódico publicado. Periódico Artigo Frequência (\%)

\begin{tabular}{|l|c|c|}
\hline AORN Journal & 06 & 54,55 \\
\hline Acta Anaesthesiologica Taiwanica & 01 & 9,09 \\
\hline Journal of Urology & 01 & 9,09 \\
\hline Perioperative Nursing Clinics & 01 & 9,09 \\
\hline Pennsylvania Nurse Journal & 01 & 9,09 \\
\hline Journal of Nursing Practice & 01 & 9,09 \\
\hline Total & 11 & 100 \\
\hline
\end{tabular}

AORN: Association of periOperative Registered Nurses.

Tabela 2. Nível de evidência dos artigos incluídos no estudo, de acordo com o Centro de Medicina Baseada em Evidências de Oxford ${ }^{10}$.

\begin{tabular}{|l|c|c|}
\hline Nível de evidência & Artigos & Frequência (\%) \\
\hline $\begin{array}{l}\text { Revisão sistemática de ensaios } \\
\text { clínicos randomizados }\end{array}$ & 01 & 9,09 \\
\hline $\begin{array}{l}\text { Ensaio clínico randomizado } \\
\text { individual }\end{array}$ & 00 & 0,0 \\
\hline $\begin{array}{l}\text { Estudos de coorte, quase } \\
\text { experimental }\end{array}$ & 00 & 0,0 \\
\hline $\begin{array}{l}\text { Série de casos, caso controle, } \\
\text { longitudinal, prospectivo, } \\
\text { retrospectivo, metodológico de } \\
\text { validação }\end{array}$ & 01 & 9,09 \\
\hline $\begin{array}{l}\text { Qualitativo, estudo de caso, } \\
\text { exploratório, quantitativo } \\
\text { descritivo }\end{array}$ & 09 & 81,82 \\
\hline \begin{tabular}{l} 
Total \\
\hline
\end{tabular} & 11 & 100 \\
\hline
\end{tabular}

artigo que respondesse à questão norteadora. Ressalta-se que, durante a busca nas bases de dados, não foram encontrados estudos publicados no idioma português, o que reforça a ideia de a temática ser recente no Brasil.

Quanto aos periódicos, o Association of periOperative Registered Nurses (AORN) Journal publicou seis artigos e as demais revistas tiveram, cada uma, apenas uma publicação. No que se refere ao método, as pesquisas que predominaram foram as de caráter qualitativo. No tocante ao nível de evidência, a maioria dos artigos foi classificado como 5, um foi classificado como 4 e outro como 2.

Não houve autor que se destacasse nas publicações da temática sobre a atuação do enfermeiro na cirurgia robótica, uma vez que todos os autores dos artigos selecionados tiveram apenas uma publicação cada um.

Atendendo ao objetivo traçado, a análise descritiva e analítica possibilitou delimitar dois tópicos, descrito na sequência.

\section{Desafios enfrentados pelo enfermeiro na cirurgia robótica}

Entre os principais desafios que apareceram nos artigos revisados, destacam-se o desenvolvimento de novas competências, a formação de equipe e a segurança do paciente em relação à cirurgia robótica.

\section{Novas competências a serem desenvolvidas pela equipe de enfermagem}

$\mathrm{O}$ avanço tecnológico visa a aumentar a eficiência em atender à demanda, aperfeiçoando os equipamentos e os instrumentais, melhorando as técnicas e os procedimentos $\left(\mathrm{A} 8^{19}\right)$. Também a cirurgia robótica traz ao enfermeiro novos desafios em seu processo de trabalho, já que todas essas evoluções exigem desenvolvimento de novas habilidades, destreza e aperfeiçoamento progressivo e contínuo.

Os enfermeiros de CC e os demais membros da equipe são fundamentais para dar continuidade aos avanços e para garantir a total segurança dos pacientes que são submetidos a esse procedimento cirúrgico $\left(\mathrm{A} 3^{14}\right)$. O papel do enfermeiro na cirurgia robótica é dinâmico e multifacetado e inclui inúmeras responsabilidades, tais como: garantia de instrumentais disponíveis, organização da equipe e segurança do paciente. É de responsabilidade do profissional da enfermagem, também, o registro de todos os materiais utilizados no procedimento $\left(\mathrm{A} 2^{13}, \mathrm{~A} 3^{14}, \mathrm{~A} 4^{15}\right)$.

Um enfermeiro atuante na cirurgia robótica pode construir uma visão abrangente de como, por que e quando um sistema 
está sendo usado. Isso permite a tomada de decisões mais precisas relacionadas a diversas competências $\left(\mathrm{A} 2^{13}\right)$. A constante presença do enfermeiro nos procedimentos também oportuniza avaliações corretas sobre as necessidades educacionais e de recursos humanos $\left(\mathrm{A} 1^{12}, \mathrm{~A} 3^{14}\right)$.

Com a participação do enfermeiro de forma dinâmica e eficaz, problemas do dia a dia, como, por exemplo, com o equipamento ou com os instrumentos do sistema robótico, podem ser resolvidos mais rapidamente e com mais precisão, trazendo melhorias e também sucesso para o programa na instituição. O sistema robótico tem a tecnologia e as capacidades de um computador voltado aos cuidados de saúde; é capaz de armazenar memória de eventos passados e erros no sistema, bem como permitir a interação ao vivo com engenheiros, técnicos e pessoal de apoio da empresa fabricante. Em caso de erro, membros da equipe podem receber ao vivo apoio técnico pelo telefone a partir desses engenheiros. O sistema atual é conectado por meio da internet, que transmite mensagens informatizadas para a sede da empresa, não importa onde a equipe cirúrgica esteja situada no mundo. E envolvido com a essa tecnologia está o enfermeiro $\left(\mathrm{A} 5^{16}\right)$.

Salienta-se que desenvolver um programa de cirurgia robótica em um hospital requer treinamento intenso dos cirurgiões e da equipe de enfermagem. Nesse processo de aprendizado e desenvolvimento, o enfermeiro tem papel de destaque, pois é o responsável por ofertar essa capacitação à equipe de enfermagem $\left(A 2^{13}, A 3^{14}, A 5^{16}, A 10^{21}\right)$.

\section{Formação de equipe}

Muitas etapas estão envolvidas na educação e no treinamento da equipe para o recebimento do robô. O processo de educação inicia-se semanas antes de o robô chegar. O cirurgião começa por um estudo em CD-ROM fornecido pela empresa representante da robótica e, em seguida, conclui um exame online $\left(\mathrm{A} 5^{16}\right)$.

Algumas barreiras na implementação de um programa de cirurgia robótica incluem a falta de tempo para os médicos se tornarem mais experientes no uso do robô, a fragilidade da educação continuada, recursos financeiros, tempo e recursos limitados para fornecer informações relevantes para o aprendizado da equipe $\left(\mathrm{A} 5^{16}, \mathrm{~A} 8^{19}\right)$. Esses são desafios enfrentados pelo enfermeiro que tem como uma de suas competências a formação e atualização dos membros da equipe de enfermagem. O profissional deve estar sempre atento à inserção nos programas, e os treinamentos devem ser fornecidos com o intuito de manter os colaboradores sempre atualizados e habilitados para sua prática. Deve existir a educação continuada e permanente para a orientação e para a formação de novos funcionários $\left(\mathrm{A}^{14}, \mathrm{~A} 6^{17}, \mathrm{~A} 10^{21}\right)$.
Considera-se que o papel do enfermeiro na formação de sua equipe deve ser ativo, motivador e proativo, capaz de desenvolver um perfil comprometido e dinâmico. É imprescindível ao enfermeiro ter bons conhecimentos de informática e de outros idiomas, preferencialmente o inglês, para acessar a literatura internacional e estar preparado a realizar treinamentos em instituições que já utilizam essa técnica em outros países ${ }^{1}$.

Ao iniciar um programa de cirurgia robótica, funcionários bem treinados são tão importantes quanto os equipamentos e os instrumentos necessários $\left(\mathrm{A} 2^{13}, \mathrm{~A} 10^{21}\right)$. A análise do progresso deve ocorrer em intervalos regulares, e uma forma de realizar essa revisão periódica é por meio de reuniões com a equipe $\left(\mathrm{A} 5^{16}\right)$.

\section{Segurança do paciente}

Criar a cultura de segurança é da responsabilidade de cada membro da equipe cirúrgica. O enfermeiro deve estar sempre atento para manter um ambiente adequado e com técnica asséptica. A enfermagem também atua muitas vezes como porta-voz do paciente, quando comparada a outros profissionais. Uma das responsabilidades do enfermeiro relacionadas com a segurança é o gerenciamento dos equipamentos e materiais para que o procedimento cirúrgico transcorra sem intercorrências $\left(A 8^{19}\right)$.

Recomenda-se que sejam utilizadas no procedimento cirúrgico listas de verificação, ou checklists, pois essa prática pode reduzir erros. Também se sugere a padronização no atendimento $\left(\mathrm{A} 3^{14}\right)$. A melhoria das práticas durante os procedimentos cirúrgicos deve ser baseada em evidências e apoiada por padrões profissionais, contribuindo para resultados positivos $\left(\mathrm{A} 6^{17}, \mathrm{~A} 8^{19}\right)$.

É imprescindível que todos os profissionais compreendam o sistema robótico, mas também que apreciem as nuanças do programa, orientações corretas no pré-operatório e orientações dos cuidados no pós-operatório $\left(\mathrm{A} 1^{12}\right)$.

Na robótica, especificamente no que diz respeito à segurança, deve-se destacar o posicionamento do paciente. A equipe é confrontada com novos posicionamentos cirúrgicos, necessitando atenção para garantir a segurança do paciente, pois a cirurgia robótica é um procedimento diferente da laparoscópica, já que muitas vezes são utilizados mais equipamentos e uma posição mais íngreme, denominada de Trendelenburg $\left(\mathrm{A} 9^{20}\right)$.

Durante os procedimentos, podem existir duas grandes categorias de lesão, que são classificadas como trauma mecânico e trauma térmico. Essas complicações ou lesões decorrentes da cirurgia não têm de ser chamadas de "eventos", pois nunca deveriam acontecer, visto que são evitáveis 
$\left(\mathrm{A} 6^{17}, \mathrm{~A} 9^{20}\right)$. Lesões associadas ao posicionamento ao longo da cirurgia são bem documentadas, por exemplo a compressão de um nervo - embora seja suave em alguns casos, pode causar problemas temporários no sistema vascular, que são possivelmente reversíveis com reperfusão. Quanto mais graves forem as compressões ou os alongamentos de um nervo, maior a possibilidade de ele conduzir a um edema endoneural, ou até mesmo à degeneração $\left(\mathrm{A} 9^{20}\right)$.

É pertinente salientar a importância de o paciente ser bem fixado na mesa cirúrgica, com faixas e coxins especiais, evitando deslizar durante a mobilização da cama cirúrgica no decorrer do procedimento $\left(A 9^{20}\right)$. É muito importante que o enfermeiro e a equipe cirúrgica tenham total domínio para executar a técnica de imobilização do paciente e saibam reconhecer como as consequências podem ser danosas, por imperícia, imprudência ou até mesmo negligência. Além da questão do posicionamento incorreto, as falhas de equipamento podem ser especialmente perigosas para a segurança do paciente durante os procedimentos, porque a complexidade e a sofisticação aumentam a possibilidade de ocorrer erros e sua não identificação precoce $\left(\mathrm{A} 6^{17}\right)$.

Em relação aos principais desafios do enfermeiro no contexto da cirurgia robótica, o Quadro 2 sintetiza os principais achados.

\section{Perspectivas e possibilidades neste novo campo de atuação para 0 enfermeiro}

No que concerne às principais perspectivas encontradas nos artigos revisados, destacam-se: futuro da cirurgia robótica, especificidade, melhorias na assistência ao paciente e educação permanente.

\section{Futuro da cirurgia robótica}

A cirurgia robótica tornou-se um padrão em grandes hospitais nos Estados Unidos e no mundo $\left(\mathrm{A} 5^{16}\right)$.

Quadro 2. Desafios enfrentados pelo enfermeiro na cirurgia robótica

\begin{tabular}{|l|c|}
\hline Desafios & \multicolumn{1}{|c|}{ Artigos } \\
\hline Novas competências & $A 1 ; A 2 ; A 3 ; A 4 ; A 5 ; A 8 ; A 10$ \\
\hline Formação de equipe & $A 1 ; A 3 ; A 5 ; A 6 ; A 8$ \\
\hline Segurança do paciente & $A 1 ; A 3 ; A 6 ; A 8 ; A 9$ \\
\hline
\end{tabular}

Em 1999, a Food and Drug Administration (FDA) aprovou o Da Vinci Surgical System ${ }^{\circledR}$, para uso nos Estados Unidos. O robô cirúrgico oferece ao cirurgião visão tridimensional e aumento da destreza, além de fornecer os benefícios da cirurgia laparoscópica para o paciente $\left(\mathrm{A} 5^{16}, \mathrm{~A} 8^{19}\right)$.

Desde então, os procedimentos cirúrgicos realizados por intermédio do sistema Da Vinci continuam a crescer. Esse sistema tem quatro braços robóticos: três braços de instrumentos e um braço endoscópico. O cirurgião senta em um console longe da cabeceira do paciente e manipula esses itens. Os braços anexam os trocateres, que são inseridos no paciente por meio de pequenas incisões. O monitor fornece ao cirurgião vista tridimensional do campo cirúrgico. O cirurgião pode aumentar em até 15 vezes a visualização do local em que está realizando o procedimento ajustando pedais no console $\left(\mathrm{A} 8^{19}, \mathrm{~A} 10^{21}\right)$.

Um dos problemas na implementação do programa são os custos, pois incluem a compra inicial (US\$ 1 milhão até US\$ 2,5 milhões), mais manutenção anual e o custo do uso limitado dos instrumentais, que são descartáveis $\left(\mathrm{A} 8^{18}, \mathrm{~A} 10^{21}\right)$. O aumento do número de procedimentos realizados usando o sistema robótico pode diminuir os custos por procedimento. Há fatores modificáveis capazes de contribuir para o aumento do volume anual de cirurgias, como aumentar o número de cirurgiões capazes de utilizar o sistema e ter uma equipe de enfermagem devidamente instruída $\left(\mathrm{A} 10^{21}\right)$.

O Quadro 3 apresenta os custos de dois procedimentos comuns em dólares.

Apesar de ser uma ferramenta cirúrgica excitante, seu custo pode ser um problema. Isso se deve ao fato de não existir concorrência no mercado. O Da Vinci Surgical System ${ }^{\circledR}$ é atualmente o único robô cirúrgico no mercado $\left(\mathrm{A} 10^{21}\right)$.

Embora o gasto inicial seja grande, um programa de robótica pode dar ao hospital vantagem competitiva, tornando-o líder em cuidados excepcionais. A aquisição da tecnologia por si só não é suficiente. Desenvolver um programa de robótica requer intenso treinamento, marketing, dedicação e paixão dos membros da equipe cirúrgica, prontos para assumir seus cuidados em nível elevado. O primeiro passo na criação de um programa de cirurgia robótica em qualquer estabelecimento é a realização de pesquisa do mercado geográfico. Isso ajuda a determinar se existe a necessidade potencial de

Quadro 3. Custos de dois procedimentos comuns (em dólares).

\begin{tabular}{|l|c|c|c|} 
Procedimento & $\begin{array}{c}\text { Custo dos instrumentais } \\
\text { Cirurgia convencional }\end{array}$ & $\begin{array}{c}\text { Custo dos instrumentais } \\
\text { Cirurgia laparoscópica }\end{array}$ & $\begin{array}{c}\text { Custo dos instrumentais } \\
\text { Cirurgia robótica }\end{array}$ \\
\hline Histerectomia & US\$ 198,00 & US $\$ 1.138,00$ & US\$2.210,00 \\
\hline Prostatectomia & US $\$ 78,00$ & US $\$ 533,00$ & US\$1.705,00 \\
\hline
\end{tabular}


cirurgias robóticas e se o programa será bem-sucedido. Se a pesquisa de mercado indica que um programa é necessário, o próximo passo importante é descobrir se os membros da equipe cirúrgica estão interessados na execução de procedimentos robóticos. Com um programa de robótica bem desenvolvido, uma instituição tem a oportunidade de um grande sucesso em todos os aspectos $\left(\mathrm{A} 5^{16}, \mathrm{~A} 11^{22}\right)$.

Os pacientes são os beneficiados com tamanha mudança na cirurgia, já que as cicatrizes menores são apenas um dos benefícios desse tipo de procedimento. Eles também relatam menos desconforto e menor período de recuperação, o que facilita o retorno, mais rápido, para atividades normais da vida diária. Apresentam menores perdas sanguíneas, o que também ajuda na promoção da recuperação mais rápida $\left(\mathrm{A} 3^{14}, \mathrm{~A} 7^{18}, \mathrm{~A} 8^{19}\right)$. Com tantas vantagens que a cirurgia assistida pelo robô promove ao paciente, acredita-se que esse será o futuro dos procedimentos operatórios em nível mundial.

\section{Campo de atuação: especificidade}

A formação é particularmente importante para os enfermeiros que atuam na cirurgia assistida pelo robô, em razão das suas múltiplas responsabilidades. Além de ter as mesmas responsabilidades em termos de gestão, como qualquer outro enfermeiro, por exemplo, o posicionamento, a segurança do paciente, a esterilidade de instrumentos e do campo cirúrgico, o profissional deve também saber trabalhar com o sistema robótico, cuidar do robô e solucionar os problemas que surgirem $\left(\mathrm{A} 4^{15}, \mathrm{~A} 10^{21}\right)$.

A atuação do enfermeiro no contexto da cirurgia robótica envolveáreas de atuação e funções específicas, conformeo Quadro 4.

Quadro 4. Funções do enfermeiro na cirurgia robótica.

\begin{tabular}{|c|c|}
\hline $\begin{array}{l}\text { Área de } \\
\text { atuação }\end{array}$ & Funções específicas \\
\hline $\begin{array}{l}\text { Prática } \\
\text { clínica }\end{array}$ & $\begin{array}{c}\text { Atua como coordenador do cuidado. } \\
\text { Fornece assistência direta ao paciente. } \\
\text { Mantém a experiência clínica e de competências } \\
\text { profissionais. } \\
\text { Desenvolve e envolve-se em competências de } \\
\text { gestão e consultoria. }\end{array}$ \\
\hline Educação & $\begin{array}{l}\text { Orienta e treina pessoal de enfermagem e } \\
\text { estudantes da saúde. } \\
\text { Educa e orienta os pacientes. } \\
\text { Ministra palestras e treinamentos. }\end{array}$ \\
\hline Gerência & $\begin{array}{l}\text { Atua como elo dentro da instituição. } \\
\text { Estabelece relacionamento com o fabricante e } \\
\text { com os fornecedores. }\end{array}$ \\
\hline Pesquisa & $\begin{array}{l}\text { Participa de coleta de dados. } \\
\text { Participa de gerenciamento de dados. } \\
\text { Assegura a divulgação de dados. }\end{array}$ \\
\hline
\end{tabular}

O enfermeiro tem o potencial de melhorar a qualidade dos cuidados de saúde, reduzindo os custos, os erros e o tempo de cirurgia; e aumentando a eficácia de gestão $\left(\mathrm{A} 3^{14}, \mathrm{~A} 7^{18}, \mathrm{~A} 10^{21}\right)$.

\section{Melhorias na assistência ao paciente}

Os avanços da tecnologia têm melhorado a cirurgia minimamente invasiva, executando a cirurgia por meio de incisões cada vez menores, já que os instrumentais próprios eliminam a necessidade de grandes incisões, promovendo ergonomia, ativação independente de energia monopolar e bipolar, menos exposição da equipe a fluidos e secreções e diminuindo as chances de contaminação, isso porque os braços robotizados executam a manipulação cirúrgica $\left(\mathrm{A} 8^{19}\right)$. Contudo, é preciso desenvolver padrões ou diretrizes clínicas para definir a ideal aplicação da cirurgia assistida por robô, de modo a garantir um padrão de atendimento que vise à segurança do paciente $\left(\mathrm{A} 8^{19}\right)$. A implementação de um caminho clínico ou diretriz guiado por prática baseada em evidências envolve mudanças. O paciente, atualmente, está mais consciente da nova tecnologia cirúrgica $\left(\mathrm{A} 7^{18}, \mathrm{~A} 8^{19}\right)$.

A literatura disponível também sugere que os pacientes se beneficiam com técnicas robóticas, mediante a diminuição de custos relacionados à internação (hotelaria) e a redução de tratamentos oriundos de infecção. O hospital beneficia-se porque os pacientes têm curta hospitalização. Por isso, um número maior de pacientes é atendido $\left(\mathrm{A} 8^{19}\right)$.

A tecnologia é cada vez mais utilizada para melhorar a qualidade dos cuidados de saúde e usá-la deverá beneficiar os pacientes em vários aspectos $\left(\mathrm{A} 4^{15}\right)$. Ressalta-se que os avanços do conhecimento e da técnica têm forte repercussão na área da saúde, tanto para diagnosticar como para tratar, e tudo isso reflete diretamente no conforto pessoal, na qualidade de vida e na longevidade das pessoas.

Entretanto, o avanço tecnológico não deve trazer aspecto frio ou mecânico, nem algo desumano na relação entre os envolvidos com o atendimento à saúde. Deve-se buscar o equilíbrio entre a sofisticação de última geração e o relacionamento humano, com a compreensão das necessidades afetivas das pessoas ${ }^{23}$.

\section{Educação permanente}

Os enfermeiros são os profissionais de cuidados de saúde selecionados para assumir o papel de coordenador e educador $\left(\mathrm{A} 1^{12}\right)$. Algumas responsabilidades importantes do enfermeiro são garantir, manter e desenvolver na equipe habilidades e competência, além de promover pesquisa baseada em evidências $\left(A 4^{15}\right)$, para certificar que os funcionários adquiram o conhecimento necessário para desempenhar suas funções 
com segurança. O enfermeiro deve fomentar o crescimento, o desenvolvimento e a autonomia de todos; ele é o responsável por assegurar que cada membro da sua equipe tenha a formação necessária. Deve, também, ser treinado em informática para melhor aplicar os conhecimentos ( $\left.\mathrm{A} 4^{15}, \mathrm{~A} 11^{22}\right)$.

A educação dos profissionais de enfermagem precisa de atenção, já que há necessidade de preparar as pessoas para as mudanças que têm ocorrido nas instituições de saúde, de modo que se conciliem as necessidades de desenvolvimento pessoal e do grupo com as necessidades da instituição e com as da sociedade ${ }^{24}$. A educação permanente é um processo que educa, possibilitando o surgimento de um espaço para pensar e fazer no trabalho, com destaque para o papel das instituições de saúde no desenvolvimento das capacidades dos profissionais, o que contribui para diversas situações de melhorias ${ }^{24,25}$.

Em relação às principais perspectivas e possibilidades para o enfermeiro no contexto da cirurgia robótica, o Quadro 5 sintetiza os principais achados.

Com o uso crescente da cirurgia robótica, há a necessidade de resultados baseados em evidências para apoiá-la $\left(A 8^{19}\right)$. O sucesso do programa robótico envolve foco no paciente, combinando segurança, recursos humanos, educação permanente, sensibilização do público e um modelo eficiente de gestão $\left(\mathrm{A} 1^{12}, \mathrm{~A} 8^{19}\right)$.

Quadro 5. Perspectivas e possibilidades para o enfermeiro na cirurgia robótica.

\begin{tabular}{|l|c|}
\hline Perspectivas & Artigos \\
\hline Futuro da cirurgia robótica & A3; A5; A7; A8; A10; A11 \\
\hline Especificidade & A3; A4; A7; A10 \\
\hline Melhorias para o paciente & A4; A7; A8 \\
\hline Educação permanente & A1; A4; A8; A11 \\
\hline
\end{tabular}

\section{CONSIDERAÇÕES FINAIS}

A cirurgia robótica é uma tecnologia crescente nos hospitais, deflagrando a necessidade de preparo, capacitação e atualização dos profissionais de saúde, especialmente do enfermeiro, para dar o suporte necessário que um programa de robótica exige.

Os aspectos discutidos neste estudo contribuem com novos saberes a respeito da cirurgia robótica e da atuação do enfermeiro, permitindo identificar os desafios que estão inseridos no programa e na atuação desses profissionais, propiciando contemplar as novas possibilidades e perspectivas e oportunizando, dessa forma, mais reflexão sobre o cenário que se apresenta.

No tocante aos desafios dos enfermeiros, identificaramse a segurança do paciente, a capacitação da equipe e novas competências a serem desenvolvidas. O enfermeiro deve ser muito mais do que um gerente nesse desafio; deve ser um líder, ou seja, um verdadeiro facilitador em seu ambiente de trabalho. Cabe a ele desenvolver suas habilidades de liderança como maneira de conseguir motivar sua equipe, desenvolvendo nos profissionais o anseio pelo conhecimento e a disponibilidade para transcender os obstáculos que surgirem com o implemento dessa nova técnica, tendo como foco de desenvolvimento principalmente o cuidado ao paciente, a fim de proporcionar total segurança em seu atendimento.

Em relação às novas possibilidades, observou-se que uma nova área de atuação está sendo desenvolvida, inovação importante para melhorias no atendimento aos pacientes, que deve ser o eixo principal de todos os esforços. Assim, é essencial a educação permanente da equipe.

Destaca-se que esta revisão integrativa não esgotou as interfaces da temática. Desse modo, sugere-se que sejam desenvolvidos novos estudos sobre a atuação do enfermeiro nos procedimentos em que se utiliza a robótica. A constatação, neste estudo, da escassa bibliografia sobre a temática no país justifica a necessidade e a importância de novos estudos.

\section{REFERÊNCIAS}

1. Sousa CS, Bispo DM, CunhaAL. Capacitação em cirurgia robóticano programa deresidênciaemenfermagemperioperatória. RevSOBECC. 2016;21(4):198-202.

2. Morin E. A cabeça bem-feita: repensar a reforma, reformar o pensamento. Rio de Janeiro: Bertrand Brasil; 2000.

3. Baggio MA, Erdmann AL, Sasso GT. Cuidado humano e tecnologia na enfermagem contemporânea e complexa. Texto Contexto Enferm. 2010;19(2):378-85.
4. Tramontini CC, Lopes DF, Kikuchi EM, Kemmer LF, Garanhani ML. Repensando a formação do gerente do processo de trabalho do enfermeiro de centro cirúrgico e centro de material. Rev SOBECC. 2002;7(1):11-5.

5. Sant'Anna RT, Prates PR, Sant'Anna JR, Prates PR, Kalil RA, Santos DE et al. Emprego de sistemas robóticos na cirurgia cardiovascular. Rev Bras Cir Cardiovasc. 2004;19(2):171-8. DOI: 10.1590/S0102-76382004000200012. 
6. Lemes C. Tempos modernos? Rev Onco [Internet]. 2011/2012 [acesso em 2014 ago. 4];9:12-6. Disponível em: https://issuu.com/ revista-onco/docs/onco-ed.09.

7. Instituto Nacional de Câncer José Alencar Gomes Da Silva (INCA). 0 futuro chega à sala de operações do INCA. Rev Rede Cancer. 2012;7:10-2.

8. Oliveira MA. Gerenciamento de novas tecnologias em centro cirúrgico pelas enfermeiras nos hospitais de Feira de Santana, BA. Rev Bras Enferm. 2004;57(3):292-7. DOI: 10.1590/S0034-71672004000300007

9. Alves AF, Grassia RC, Carvalho R. Cirurgia robótica de revascularização miocárdica: em busca de evidência científica no uso da tecnologia do século XXI. Rev SOBECC. 2012;17(3):31-42.

10. Oxford Centre for Evidence-Based Medicine (UK). Levels of Evidence Working Group [Internet]. Oxford: CEBM; 2011 [acesso em 2017 Jan. 13]. Disponível em: http://www.cebm.net/wp-content/uploads/2014/06/ CEBM-Levels-of-Evidence-2.1.pdf.

11. Brasil. Lei n० 9.610 , de 19 de fevereiro de 1998. Altera, atualiza e consolida a legislação sobre direitos autorais e dá outras providências. Brasília; 1998.

12. Schmock BA. Technological advances in the operating room. Pa Nurse. 2009;64(1):13-28.

13. Booth BE. Robotics in nursing. J Pract Nurs. 2011;61(4):12-3.

14. Ulmer BC. Best practices for minimally invasive procedures. AORN J. 2010;91(5):558-72. DOI: 10.1016/j.aorn.2009.12.028

15. Sweeney $P$. The effects of information technology on perioperative nursing. AORN J. 2010;92(5):528-40. DOI: 10.1016/j.aorn.2010.02.016

16. Zender J, Thell C. Developing a successful robotic surgery program in a rural hospital. AORN J. 2010;92(1):72-83. DOl: 10.1016/j.aorn.2009.10.024
17. Stanton C. Keeping up with technology. AORN J. 2011;93(1):8-9.

18. Thomas CC. Role of the perioperative nurse in robotic surgery. Perioper Nurs Clin. 2011;6(3):227-34. DOI: 10.1016/j.cpen.2011.06.005

19. Walters L, Eley S. Robotica-assisted surgery and the need for standardized pathways and clinical guidelines. AORN J. 2011;93(4):45563. DOI: 10.1016/j.aorn.2010.05.032

20. Mills JT, Burris MB, Warburton DJ, Conaway MR, Schenkman NS, Krupski TL. Positioning injuries associated with robotic assisted urological surgery. J Urol. 2013;190(2):580-4. DOI: 10.1016/j. juro.2013.02.3185

21. Nayeemuddin M, Daley SC, Ellsworth P. Modifiable factors to decrease the cost of robotic-assisted procedures. AORN J. 2013;98(4):343-52. DOI: 10.1016/j.aorn.2013.08.012

22. Lee CC, Lin SP, Yang SL, Tsou MY, Chang KY. Evaluating the influence of perceived organizational learning capability on user acceptance of information technology among operating room nurse staff. Acta Anaesthesiol Taiwan. 2013;51(1):22-7. DOI: 10.1016/j.aat.2013.03.013

23. Ballone GJ. Humanização do atendimento em saúde. Psiq Web [Internet]; 2008 [acesso em 2017 Jun. 10]. Disponível em: http:// www.psiqweb.med.br/site/?area=NO/LerNoticia\&idNoticia=251.

24. Paschoal AS, Montovani MF, Méier MJ. Percepção da educação permanente, continuada e em serviço para enfermeiros de um hospital de ensino. Rev Esc Enferm. 2007;41 (3):478-84. DOI: 10.1590/ S0080-62342007000300019

25. Amestoy SC, Schveitzer MC, Meirelles BH, Backes VM, Erdmann AL. Paralelo entre educação permanente em saúde e administração complexa. Rev Gaúcha Enferm. 2010;31(2):383-7. DOI: 10.1590/ S1983-14472010000200025 